1 Universidade Estadual do Oeste do Paraná (Unioeste) - Cascavel (PR), Brasil.

brunabratti@gmail.com

2 Universidade Estadual do Oeste do Paraná

(Unioeste) - Cascavel (PR), Brasil.

clausviera@hotmail.com

3 Universidade Estadual do Oeste do Paraná

(Unioeste) - Cascavel (PR), Brasil.

claudiross@gmail.com

4 Universidade Estadual do Oeste do Paraná

(Unioeste) - Cascavel (PR), Brasil.

phallcha@terra.com.br

5 Universidade Estadual do Oeste do Paraná (Unioeste) - Cascavel (PR), Brasil.

beatriz.oliveira@unioeste.br

\section{Avaliação da longitudinalidade em unidades de Atenção Primária à Saúde}

\author{
Evaluation of the longitudinality in Primary Health Care units
}

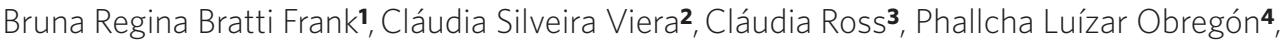 \\ Beatriz Rosana Gonçalves de Oliveira Toso ${ }^{\mathbf{5}}$
}

RESUMO Estudo para avaliar a efetividade da Atenção Primária à Saúde direcionada a crianças, por meio do atributo longitudinalidade, em unidades de saúde com e sem Estratégia Saúde da Família em município do Oeste do Paraná. Pesquisa quantitativa, descritiva, com utilização do instrumento de coleta de dados PCATool-Brasil, versão criança, aplicado a 61 familiares de crianças menores de 12 anos. A longitudinalidade foi mais bem avaliada na Unidade Básica de Saúde no tocante à saúde da família, demonstrando a necessidade de mudanças profundas no processo de trabalho das Unidades de Saúde da Família para melhoria da qualidade da atenção e efetiva implementação das diretrizes propostas pela Estratégia.

PALAVRAS-CHAVE Atenção Primária à Saúde; Saúde da criança; Continuidade da assistência ao paciente.

ABSTRACT Study to evaluate the effectiveness of Primary Health Care directed to children through the longitudinality attribute, in health units with and without Family Health Strategy in a western municipality of Paraná State. Descriptive quantitative, research, with the use of the data collection instrument PCATool - Brazil, child version, applied to 61 family members of children under 12 years old. The longitudinality was best evaluated in the health unit with regard to the family health, demonstrating the relevance of deep changes in the work process in the Family Health Units, for the improvement of the attention quality and an effective deployment of the proposed guidelines by the Strategy.

KEYWORDS Primary Health Care; Child health; Continuity of patient care. 


\section{Introdução}

De acordo com a Comissão Conjunta de Acreditação de Organizações de Saúde (JCAHO), a efetividade é definida como o grau com que uma determinada intervenção beneficia a população definida, sob condições regulares de uso. Segundo o Comitê de Qualidade da Atenção Primária na América, a efetividade constitui um atributo do cuidado em saúde que se baseia no uso de evidências adquiridas para determinar se uma intervenção produz melhores resultados do que outras possibilidades de ação. Em síntese, a efetividade é o grau com que a assistência, os serviços e as ações prestadas atingem os resultados esperados (VIACAVA, ET AL. 2012).

A efetividade da Atenção Primária à Saúde (APS) é capaz de assegurar à população a universalização do acesso aos serviços de saúde, promovendo ações de prevenção de doenças e promoção da saúde. A APS é considerada o primeiro ponto de contato do indivíduo com o sistema de saúde. Por esse motivo, é necessário que a atenção seja de fácil acesso a toda a população. A Atenção Básica como porta de entrada encontra referência na Carta dos Direitos dos Usuários do Sistema Único de Saúde (BRASIL, 2006), que assegura aos cidadãos acesso de forma ordenada e organizada aos sistemas de saúde, prioritariamente, por meio dos serviços de atenção primária encontrados em locais próximos à moradia dos usuários (STARFIELD, 2002).

A APS busca implementar outros modelos de assistência à saúde, considerando que o modelo hospitalocêntrico não atende às transformações do mundo moderno e às necessidades de saúde da população e de suas famílias. A frequente busca de mudanças no modelo de atenção à saúde resultou, em 1994, na criação do Programa Saúde da Família (PSF), mais tarde designado Estratégia Saúde da Família (ESF), por se tratar de uma estratégia operacional do governo para implementar a APS no País (ROECHER; BUDÓ; MARCON, 2012).
A APS abrange quatro atributos essenciais: a acessibilidade, a longitudinalidade, a integralidade e a coordenação; e três atributos derivados: a orientação comunitária, a orientação familiar e a competência cultural. Entre os atributos essenciais para a APS e, consequentemente, para a ESF, destaca-se a longitudinalidade, que tem visa o acompanhamento dos usuários pela equipe de saúde, promovendo uma assistência continuada ao longo do tempo (STARFIELD, 2002). A continuidade não é obrigatória para que a relação exista, pois interrupções na continuidade não interrompem a relação.

Os serviços de APS são considerados ambientes preferenciais para a promoção da saúde infantil, e foi a partir do atributo longitudinalidade que a pesquisa avaliou a efetividade da APS em unidades de saúde com e sem ESF, influenciando na saúde da criança em município do Oeste do Paraná.

\section{Metodologia}

Trata-se de pesquisa de avaliação dos modelos de APS (unidade de saúde com e sem ESF) relativa à extensão do atributo longitudinalidade nos cuidados primários à saúde da criança, em município do Oeste do Paraná, utilizando a abordagem quantitativa.

No momento da pesquisa, o município em estudo apresentava apenas uma unidade de saúde com ESF na região urbana, que foi selecionada para a pesquisa. Já para a escolha de uma representante entre as 22 Unidades Básicas de Saúde (UBS) sem ESF existentes no momento da coleta de dados, efetuou-se um sorteio.

Assim, a população do estudo foi composta de familiares e/ou cuidadores/representantes legais de crianças com idade até doze anos incompletos, atendidas por médicos e/ ou enfermeiros nas unidades selecionadas em um período de seis meses anteriores à etapa de coleta de dados, totalizando 2.773 atendimentos para a UBS sem ESF e 1.092 
para a UBS com ESF. A partir desses dados, foi estimado o tamanho da amostra nos serviços elencados por amostragem probabilística casual simples estratificada, com partilha proporcional por unidade, resultando em 44 sujeitos da UBS com ESF e 17 da UBS sem ESF, totalizando 61 sujeitos (responsáveis das crianças), selecionados por amostragem sistemática na fila de espera para consulta médica ou puericultura.

A coleta de dados se deu por meio do Instrumento de Avaliação da Atenção Primária denominado PCATool (Primary Care Assessment Tool), versão criança, validado no Brasil em 2010 (BRASIL, 2010).

Os critérios de inclusão da amostra foram: ser residente em área urbana do município; ter condições para ser o respondente do formulário, tais como capacidade de entendimento, expressão e compreensão dos documentos apresentados (Termo de Consentimento Livre e Esclarecido); o acompanhante da criança na unidade deveria ser seu principal cuidador; idade da criança até doze anos; o cuidador/respondente deveria conhecer a unidade que iria avaliar, sendo assim, foi incluído aquele que já havia levado a criança para atendimento naquela unidade pelo menos duas vezes antes da abordagem; apenas respondentes moradores da região de abrangência da unidade; e, ainda, moradores respondentes residentes fora da territorialização, mas que utilizavam o serviço por opção.

As variáveis analisadas relativas ao atributo longitudinalidade, um dos componentes do formulário de avaliação PCATool, foram: D1 - Regularidade de atendimento pelo profissional; D2 - Possibilidade de tirar dúvidas pelo telefone; D3 - Entendimento do profissional sobre as perguntas dos responsáveis/ principais cuidadores; D4 - Entendimento da resposta do profissional por parte dos responsáveis/principais cuidadores; D5 - Disponibilidade de tempo para o responsável/principal cuidador expor as preocupações e os problemas com relação à saúde da criança; D6 - Responsável/principal cuidador se sente livre para expor suas preocupações e seus problemas com relação à saúde da criança; D7 - Profissional conhece a criança como pessoa ou como problema de saúde; D8 - Profissional conhece a história completa da criança; D9 - Profissional conhece os medicamentos que a criança toma/tomou; D10 - O responsável/principal cuidador mudaria de serviço de saúde, mesmo que isso fosse muito fácil; D11 - Profissional conhece a família bastante bem; D12 - O profissional de saúde sabe quais são os problemas mais importantes para o responsável/principal cuidador e sua família; D13 - O profissional de saúde sabe sobre o trabalho ou emprego dos familiares da criança; D14 - O profissional saberia de alguma forma se o responsável/ principal cuidador tivesse problemas para obter ou pagar pelos medicamentos de que sua criança precisa. De acordo com cada variável, têm-se as seguintes respostas: Certo que não, Provável que não, Provável que sim, Certo que sim, Não sei/Não lembro.

Os dados foram analisados quantitativamente sob a forma de estatística descritiva de distribuição de frequências absolutas e relativas, médias e medianas, sendo apresentados em forma de tabela para posterior comparação com a literatura disponível acerca da temática em questão.

A pesquisa desenvolveu-se de acordo com as normas da Resolução 196/96, do Conselho Nacional de Saúde, e o projeto foi aprovado por Comitê de Ética em Pesquisa com seres humanos sob parecer $\mathrm{n}^{\circ}$ 044/2012-CEP. Em dezembro de 2012, entrou em vigor a Resolução 466/12, sendo que os preceitos éticos em questão foram mantidos.

\section{Resultados e discussão}

Os resultados da avaliação dos modelos de APS, de acordo com a opinião dos responsáveis/representantes legais das crianças, são apresentados na tabela 1 a seguir. 
Tabela 1. Avaliação do atributo longitudinalidade em Unidade Básica de Saúde e Unidade de Saúde da Família (USF) de acordo com os responsáveis de crianças atendidas em 2012/2013

\begin{tabular}{|c|c|c|c|c|}
\hline \multirow[t]{3}{*}{ Itens avaliados } & \multicolumn{4}{|c|}{ Unidades de Saúde } \\
\hline & \multicolumn{2}{|c|}{ USF } & \multicolumn{2}{|c|}{ UBS } \\
\hline & $\mathbf{N}$ & $\%$ & $\mathbf{N}$ & $\%$ \\
\hline \multicolumn{5}{|c|}{ D1 - Regularidade do atendimento pelo profissional } \\
\hline Certo que não & 0 & 0,0 & 9 & 20,4 \\
\hline Provável que não & 2 & 11,7 & 1 & 2,3 \\
\hline Provável que sim & 3 & 17,6 & 3 & 6,9 \\
\hline Certo que sim & 12 & 70,6 & 30 & 68,2 \\
\hline Não sei/Não lembro & 0 & 0,0 & 1 & 2,3 \\
\hline Total & 17 & 100 & 44 & 100 \\
\hline \multicolumn{5}{|c|}{ D2 - Possibilidade de tirar dúvidas pelo telefone } \\
\hline Certo que não & 7 & 41,1 & 18 & 40,9 \\
\hline Provável que não & 0 & 0,0 & 3 & 6,9 \\
\hline Provável que sim & 1 & 5,8 & 5 & 11,3 \\
\hline Certo que sim & 6 & 35,1 & 9 & 20,4 \\
\hline Não sei/Não lembro & 3 & 17,5 & 9 & 20,4 \\
\hline Total & 17 & 100 & 44 & 100 \\
\hline \multicolumn{5}{|c|}{ D3 - Entendimento do profissional sobre as perguntas do responsável } \\
\hline Certo que não & 1 & 5,8 & 1 & 2,3 \\
\hline Provável que não & 1 & 5,8 & 1 & 2,3 \\
\hline Provável que sim & 1 & 5,8 & 2 & 4,5 \\
\hline Certo que sim & 14 & 85,3 & 39 & 88,6 \\
\hline Não sei/Não lembro & 0 & 0,0 & 1 & 2,3 \\
\hline Total & 17 & 100 & 44 & 100 \\
\hline \multicolumn{5}{|c|}{ D4 - Entendimento da resposta do profissional } \\
\hline Certo que não & 2 & 11,6 & 0 & 0,0 \\
\hline Provável que não & 1 & 5,8 & 0 & 0,0 \\
\hline Provável que sim & 1 & 5,8 & 2 & 4,5 \\
\hline Certo que sim & 13 & 76,4 & 42 & 95,4 \\
\hline Não sei/Não lembro & 0 & 0,0 & 0 & 0,0 \\
\hline Total & 17 & 100 & 44 & 100 \\
\hline \multicolumn{5}{|c|}{ D5 - Tempo para o responsável expor as preocupações e os problemas } \\
\hline Certo que não & 2 & 11,6 & 1 & 2,3 \\
\hline Provável que não & 1 & 5,8 & 4 & 9,1 \\
\hline Provável que sim & 2 & 11,6 & 0 & 0,0 \\
\hline Certo que sim & 12 & 70,5 & 39 & 88,6 \\
\hline Não sei/Não lembro & 0 & 0,0 & 0 & 0,0 \\
\hline Total & 17 & 100 & 44 & 100 \\
\hline
\end{tabular}


Tabela 1. (cont.)

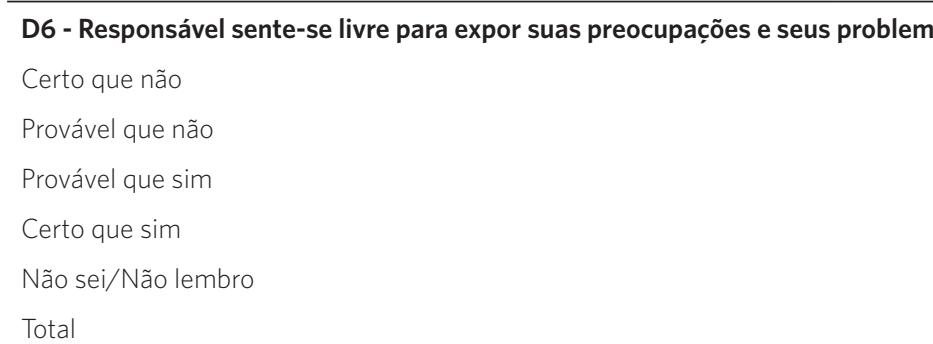

D7 - Profissional conhece criança como pessoa ou como problema de saúde

Certo que não

Provável que não

Provável que sim

Certo que sim

Não sei/Não lembro

Total

D8 - Profissional conhece história completa da criança

Certo que não

Provável que não

Provável que sim

Certo que sim

Não sei/Não lembro

Total

D9 - Profissional conhece os medicamentos que a criança toma/tomou

Certo que não

Provável que não

Provável que sim

Certo que sim

Não sei/Não lembro

Total

D10 - Responsável mudaria de serviço de saúde

Certo que não

Provável que não

Provável que sim

Certo que sim

Não sei/Não lembro

Total

D11 - O profissional conhece a família bastante bem

Certo que não

Provável que não

Provável que sim

Certo que sim

Não sei/Não lembro

Total

$\begin{array}{rrrr}3 & 17,5 & 3 & 6,8 \\ 2 & 11,6 & 1 & 2,3 \\ 1 & 5,8 & 1 & 2,3 \\ 11 & 64,5 & 39 & 88,6 \\ 0 & 0,0 & 0 & 0,0 \\ 17 & 100 & 44 & 100\end{array}$

$\begin{array}{llll}6 & 35,1 & 14 & 31,8\end{array}$

$5 \quad 294$

5,8

29,4

0,0

100

$6 \quad 13,6$

36,8

$\begin{array}{llll}5 & 29,4 & 19 & 43,2\end{array}$

$\begin{array}{llll}0 & 0,0 & 2 & 4,5\end{array}$

17

44

100

$\begin{array}{rrrr}3 & 17,5 & 8 & 18,2 \\ 1 & 5,8 & 2 & 4,5 \\ 1 & 5,8 & 2 & 4,5 \\ 12 & 70,4 & 31 & 70,4 \\ 0 & 0,0 & 1 & 2,3 \\ 17 & 100 & 44 & 100\end{array}$

100

100

$\begin{array}{rrrr}2 & 11,6 & 14 & 31,8 \\ 3 & 17,5 & 0 & 0,0 \\ 2 & 11,6 & 6 & 13,6 \\ 10 & 58,7 & 24 & 54,5 \\ 0 & 0,0 & 0 & 0,0 \\ 17 & 100 & 44 & 100\end{array}$

\begin{tabular}{rrrr}
5 & 29,4 & 22 & 50,0 \\
3 & 17,5 & 2 & 4,5 \\
4 & 23,4 & 2 & 4,5 \\
5 & 29,4 & 16 & 36,3 \\
0 & 0,0 & 2 & 4,5 \\
17 & 100 & 44 & 100 \\
& & & \\
10 & 58,7 & 23 & 52,3 \\
3 & 17,5 & 7 & 15,9 \\
2 & 11,6 & 3 & 6,8 \\
2 & 11,6 & 11 & 25,0 \\
0 & 0,0 & 0 & 0,0 \\
17 & 100 & 44 & 100 \\
\hline
\end{tabular}




\begin{tabular}{|c|c|c|c|c|}
\hline \multicolumn{5}{|c|}{ D12 - O profissional sabe quais são os problemas mais importantes para o responsável } \\
\hline Certo que não & 12 & 70,4 & 21 & 47,7 \\
\hline Provável que não & 2 & 11,6 & 5 & 11,3 \\
\hline Provável que sim & 2 & 11,6 & 5 & 11,3 \\
\hline Claro que sim & 1 & 5,8 & 12 & 27,3 \\
\hline Não sei/Não lembro & 0 & 0,0 & 1 & 2,3 \\
\hline Total & 17 & 100 & 44 & 100 \\
\hline \multicolumn{5}{|c|}{ D13 - Profissional de saúde sabe sobre o trabalho ou emprego dos familiares } \\
\hline Certo que não & 13 & 76,4 & 29 & 65,9 \\
\hline Provável que não & 0 & 0,0 & 2 & 4,5 \\
\hline Provável que sim & 0 & 0,0 & 4 & 9,1 \\
\hline Certo que sim & 2 & 11,6 & 6 & 13,6 \\
\hline Não sei/Não lembro & 2 & 11,6 & 3 & 6,8 \\
\hline Total & 17 & 100 & 44 & 100 \\
\hline \multicolumn{5}{|c|}{$\begin{array}{l}\text { D14 Profissional saberia se o responsável tivesse problemas para pagar pelos } \\
\text { medicamentos }\end{array}$} \\
\hline Certo que não & 14 & 82,3 & 22 & 50,0 \\
\hline Provável que não & 0 & 0,0 & 0 & 0,0 \\
\hline Provável que sim & 1 & 5,8 & 7 & 15,9 \\
\hline Certo que sim & 1 & 5,8 & 15 & 34,1 \\
\hline Não sei/Não lembro & 1 & 5,8 & 0 & 0,0 \\
\hline Total & 17 & 100 & 44 & 100 \\
\hline
\end{tabular}

Fonte: Instrumento de Avaliação da Atenção Primária (PCATool-Brasil), versão criança: atributo longitudinalidade (BRASIL, 2010)

A avaliação da qualidade da longitudinalidade na APS baseia-se em mensurar a capacidade dos usuários de reconhecer sua fonte de atenção primária, sendo que esta deveria ser capaz de identificar sua população assistida. Além disso, os usuários utilizariam essa fonte habitual para todos os problemas, com exceção daqueles que exigem encaminhamento médico por seu maior grau de complexidade (STARFIELD, 2002).

A pesquisa, nesse sentido, buscou identificar a opinião dos responsáveis/principais cuidadores das crianças, usuários de dois modelos de APS, com a finalidade de reconhecer os pontos positivos e as dificuldades existentes quanto ao atributo longitudinalidade.

Os resultados positivos encontrados no tocante à regularidade de atendimento pelo mesmo profissional (D1), em ambos os modelos, sugerem o estabelecimento de um forte vínculo com as unidades em questão (70,6\% na UBS com ESF e $68,2 \%$ na UBS sem ESF). Tal fato demonstra que esses usuários reconhecem as UBS como uma fonte regular de cuidados, significando que a população tem esses serviços como referência habitual para suas necessidades de saúde. Sendo assim, como pontua Starfield (2002), o acompanhamento do usuário por essa fonte regular, ao longo do tempo, traduz uma relação de responsabilidade, vínculo e confiança na equipe de saúde, possibilitando diagnósticos mais precisos e tratamentos mais eficazes, promovendo maior resolutividade dos problemas de saúde da população.

Esse vínculo entre o serviço e a comunidade é evidenciado em outro dado apontado na 
pesquisa, que revela que em 7 (41,1\%) casos de UBS com ESF e em 18 (40,9\%) de UBS sem ESF, os responsáveis não esclarecem dúvidas sobre o tratamento da criança pelo telefone (D2). Uma possível explicação para esse fato pode advir do desconhecimento de cuidadores e responsáveis das crianças dos serviços e ações oferecidos na UBS com ESF, como, por exemplo, o sistema de telefonia, presente em algumas unidades. Contudo, essa realidade não é comum em todas as regiões do Brasil, já que muitas vezes esse tipo de serviço não está disponível. Para além desses aspectos, tem-se ainda a fragilidade do vínculo entre profissional e usuário, o que dificulta o estabelecimento de uma relação pessoal ao longo do tempo, a qual permitiria aos profissionais conhecer os pacientes e vice-versa, implicando, possivelmente, em menor número de consultas, pois muitos problemas poderiam ser resolvidos pelo telefone (STARFIELD, 2002).

As questões relacionadas à comunicação profissional/usuário demonstram resultados satisfatórios para ambos os serviços. A pesquisa encontrou que em 14 (85,3\%) casos na UBS com ESF e em 39 (88,6\%) na UBS sem ESF, o profissional de saúde entende o que o responsável pergunta (D3), da mesma forma que há entendimento por parte dos responsáveis acerca das respostas do profissional $(76,4 \%$ para UBS com ESF e $95,4 \%$ para UBS sem ESF) (D4). Ainda foi possível observar que o responsável tem tempo suficiente para expor suas preocupações e seus problemas (70,5\% para UBS com ESF e 88,6\% para UBS sem ESF) (D5) e que sente-se livre para expô-los ao profissional (64,5\% para UBS com ESF e 88,5\% para UBS sem ESF) (D6).

Apreende-se que a longitudinalidade refere-se ao atendimento em saúde de uma determinada equipe ao paciente, acolhendo-o de forma holística por anos. Para Baratieri, Mandu e Marcon (2012), isso resulta na promoção de familiaridade e confiança entre as partes, e essa interação se constrói especialmente pelo diálogo. Isso não significa a resolução completa dos problemas de saúde infantil, porém, o envolvimento da escuta, a valorização das queixas, a identificação das necessidades, a fim de acolher, orientar ou direcionar o atendimento para serviços que exijam maior complexidade (RIBEIRO; ROCHA; JORGE, 2010), são aspectos imprescindíveis para o atendimento humanizado e que contribuem para a resolutividade dos problemas de saúde infantil.

Segundo Mello et al. (2012), é de extrema importância proporcionar oportunidade às mães/principais cuidadores de falarem sobre seus medos, dificuldades, desejos e interesses, assim como contribuir para que conheçam seus filhos e compreendam suas necessidades, atentar-se às singularidades de cada família, promover o seguimento da criança, fortificando o vínculo profissional-usuário, e preparar as famílias para o cuidado de si e da criança.

Como já é conhecida, a atenção primária é aquele nível de atenção que oferece a entrada no sistema para todas as novas necessidades e problemas, fornece atenção sobre o indivíduo (não apenas direcionada para a enfermidade) no decorrer do tempo, para todas as condições, exceto as muito incomuns ou raras, e coordena ou integra a atenção fornecida em outro lugar ou por terceiros (BOSI; MERCADO, 2004 APUD GOMES; SILVA, 2011). Porém, os dados apresentados com relação ao reconhecimento da criança, por parte do profissional, como pessoa e não como apenas um problema de saúde (D7), demonstram que, para os serviços avaliados, essa atenção sobre o indivíduo não ocorre de forma coerente com os princípios da APS. E, nesse caso, observa-se, ainda, uma discrepância significativa entre os serviços. Para a UBS com ESF, 11 (64,5\%) dos responsáveis afirmam que o profissional conhece a criança mais como um problema de saúde. Já para UBS sem ESF, 22 (50\%) declaram que o profissional conhece a criança mais como indivíduo/pessoa, respeitando suas condições e sua totalidade. 
Os dados apresentados pela unidade com ESF mostram-se diferentes do esperado para o modelo, refletindo a hegemonia do modelo de atenção biomédico e curativo, característico dos serviços de saúde sem ESF em nosso País. Esse aspecto demonstra que o modelo de ESF está indo de encontro às diretrizes propostas para a estratégia, de atenção integral ao usuário em seu contexto social e familiar. A substituição desse modelo por um baseado na focalização do indivíduo e da família como centro da atenção é uma das premissas da reestruturação da APS.

Segundo Starfield (2002), uma das principais dificuldades da resolutividade dos serviços prestados pela APS encontra-se na postura dos profissionais frente aos usuários dos serviços, muitas vezes direcionando as atividades assistenciais para a doença e não para a pessoa, ao número de atendimentos e não para a qualidade da atenção, impedindo a formação de vínculos e serviço integral.

Ainda, a estabilidade dos profissionais nas UBS com ESF deveria garantir maior número de desfechos nos tratamentos instituídos, melhoria da compreensão dos profissionais ao avaliar as necessidades de uma pessoa, além de proporcionar maior satisfação dos usuários durante os atendimentos, pela consolidação da relação médico-paciente (CUNHA; GIOVANELLA, 2011).

Essa característica constitui-se como elemento fundamental do modelo direcionado à saúde da família, a qual não foi identificada nesta pesquisa. A alta rotatividade de profissionais é considerada um dos aspectos críticos para o sucesso do modelo de ESF, pois pode comprometer a efetividade do serviço, prejudicando a qualidade da assistência e a satisfação do usuário (GIOVANI; VIEIRA, 2013). Nesta pesquisa, esse argumento pode ter contribuído para os dados apresentados pela ESF, visto que, no município em questão, a carga horária dos trabalhadores que atuam no serviço é diferente da dos trabalhadores das unidades sem ESF, acarretando uma maior rotatividade dos profissionais e, por conseguinte, uma maior fragilidade do vínculo com o usuário.

Por outro lado, a pesquisa demonstra que $12(70,4 \%)$ dos responsáveis/principais cuidadores na UBS com ESF certificam-se de que os profissionais têm conhecimento a respeito da história clínica completa das crianças (D8). Da mesma forma 10 (58,7\%) afirmam que os profissionais sabem quais medicamentos a criança toma/tomou durante os últimos meses (D9). Valores similares foram verificados para a UBS sem ESF: $31(70,4 \%)$ e $24(54,5 \%)$, respectivamente. A interação entre o profissional de saúde e o cuidador/responsável e a história de vida da criança é aspecto essencial para um atendimento de qualidade (BRAZ, ET AL., 2013). Nesse sentido, pode-se inferir que em ambos os modelos de atenção ocorre a valorização da história clínica dos usuários para o planejamento do cuidado.

Nesses casos, salienta-se o papel que a APS desempenha na coordenação e na organização dos fluxos de assistência, em que se observa um sistema de referência e contrarreferência dos próprios profissionais dentro do estabelecimento, mesmo tendo em vista a alta rotatividade, mantendo as informações a respeito do atendimento prestado juntamente ao sistema de informações relativo a cada usuário, ou seja, em seu prontuário (MENDES, 2011).

Quando se analisou se o responsável/ principal cuidador mudaria de serviço de saúde (D10), observou-se, novamente, avaliação positiva por parte da UBS sem ESF, em que 22 (50\%) dos usuários não mudariam de serviço. Já na UBS com ESF, poucos afirmaram que não mudariam de serviço, correspondendo a apenas $5(29,4 \%)$ sujeitos.

Baratieri e Marcon (2012) afirmam que, quando os vínculos entre população e profissionais são estabelecidos, o acompanhamento ao longo do tempo é favorecido, levando a população a identificar na UBS com ESF sua fonte regular de cuidados. Contudo, muitas vezes, o usuário não tem opção de mudar de 
serviço, pois encontra barreiras no acesso (SILVA, ET AL., 2013).

Constituindo-se a ESF em modelo que visa a reorganizar a produção dos cuidados de saúde, reorientando a prática assistencial para uma assistência voltada essencialmente à família, em seu contexto físico e social (ROECHER; BUDÓ; MARCON, 2012), Os valores significativamente baixos apresentados pela UBS com ESF sugerem que essa fonte regular de cuidados à saúde da criança não está fornecendo atenção em conformidade com os atributos essenciais da APS, gerando insatisfação na população adscrita.

Resultados negativos foram observados em ambos os serviços para os aspectos D11, D12, D13 e D14. Os dados apresentaram diferenças entre as unidades, sendo que a UBS com ESF apresentou os valores mais baixos. A pesquisa demonstra que os profissionais não conhecem bem a família da criança: 10 (58,7\%) na UBS com ESF e 23 (52,3\%) na UBS sem ESF), assim como, respectivamente, $12(70,4 \%)$ e $21(47,7 \%)$ não sabem quais são seus problemas mais importantes, nem sobre o emprego/trabalho dos familiares (76,4\% na UBS com ESF e 65,9\% na UBS sem ESF) e, ainda, não saberiam se a família tivesse problemas em pagar pelos medicamentos de que a criança precisa (82,3\% na UBS com ESF e $50,0 \%$ na UBS sem ESF).

Os baixos valores atribuídos indicam que, apesar de a avaliação da longitudinalidade ter sido positiva, até então, pode-se afirmar que as práticas de saúde voltadas ao conhecimento do contexto familiar ainda são frágeis e reforçam o modelo assistencial biomédico, principalmente na UBS com ESF. Diante disso, observa-se a necessidade de estreitar a relação e a consequente aproximação entre profissional e população adscrita, para possibilitar maior conhecimento, por parte dos profissionais, sobre os problemas de seus usuários, contribuindo para maior satisfação e resolutividade das necessidades da população.
Baratieri e Marcon (2012) destacam que conhecer quais são as dificuldades envolvidas na realização da atenção longitudinal e os elementos propulsores de mudanças é fundamental para que a ESF possa compreender a importância da longitudinalidade, as formas para realizá-la e melhorá-la no cotidiano profissional.

Os dados apresentados neste estudo com relação à longitudinalidade vão ao encontro do argumento de Costa et al. (2011), quando mencionam que a ESF não tem conseguido funcionar como porta de entrada do sistema de saúde, e, sim, como mais uma opção de atendimento ao usuário, o que faz prevalecer o modelo centrado no procedimento e na atenção médica. Dessa forma, não é possível observar o desenvolvimento do papel da APS em sua total abrangência, ou seja, ela não se configura como estratégia efetiva na busca de maior promoção da saúde, prevenção de doenças, melhor qualidade de saúde das crianças e maior satisfação dos cuidadores (STARFIELD, 2002).

O modo como a ESF tem sido implementada em várias regiões do País tem se mostrado pouco efetivo, uma vez que, sem modificar a organização do processo de trabalho e o foco do cuidado que deve passar da doença para o usuário e sua família, pautado no trabalho multiprofissional e nas ações de educação, prevenção e promoção de saúde, torna o serviço de saúde fragmentado e com baixa resolubilidade, o que acaba por inviabilizar a ESF como proposta de fortalecimento da APS (STARFIELD, 2002).

\section{Considerações finais}

A partir dos resultados, identificou-se que, para os cuidadores, a longitudinalidade do cuidado na UBS sem ESF foi mais bem avaliada em relação à UBS com ESF, fato que pode ter como uma de suas razões a não ocorrência de mudanças no modelo de 
atenção, apesar de a unidade ter passado a ser ESF. Assim como a maior rotatividade de profissionais nesse serviço dificulta o vínculo com a população ou, ainda, que o modo de cuidar na ESF vá de encontro ao que se passa no imaginário da população e, muitas vezes, do próprio profissional de saúde, os quais podem considerar que não ter mais na unidade de saúde o especialista constitui uma perda para o atendimento à saúde. Como o processo de implementação da ESF no município ainda está em fase inicial, é evidente que a avaliação realizada não apresente dados tão elevados, relativos a uma melhor qualidade do cuidado, em relação ao modelo da UBS sem ESF.

Diante dos resultado, identifica-se a necessidade de mudanças profundas no processo de trabalho das unidades de saúde com ESF, para melhoria da qualidade da atenção e efetiva implementação das diretrizes propostas na Estratégia.

\section{Referências}

BARATIERI, T.; MANDU, E. N. T.; MARCON, S. S. Longitudinalidade no trabalho do enfermeiro: relatos da experiência profissional. Rev. Esc. Enferm. USP, v. 46, n. 5, p. 1260-1267, 2012.

BARATIERI, T.; MARCON, S. S. Longitudinalidade no trabalho do enfermeiro: Identificando dificuldades e perspectivas de transformações. Texto Contexto Enfermagem, Florianópolis, v. 21, n.3, jul./set. 2012, p.549-57. Disponível em: <http://www.scielo.br/pdf/ tce/v2ln3/v2ln3a09.pdf >. Acesso em: 21 jan. 2013.

BRAZ, J. C. et al. A longitudinalidade e a integralidade no cuidado às crianças menores de um ano: avaliação de cuidadores. Medicina, Ribeirão Preto, v. 46, n. 4, p. 416-23, 2013. Disponível em: <http://revista.fmrp.usp.
Ainda há muito a se produzir com o propósito de avaliar os serviços de saúde em território nacional, inclusive para proporcionar comparações regionais entre os desempenhos da APS, tendo como finalidade aperfeiçoar os atendimentos prestados em todo o País. Sendo assim, enfatiza-se a utilização do PCATool como ferramenta efetiva para avaliar os serviços de APS, uma vez que contempla todos os seus atributos, possibilitando identificar a extensão destes nos serviços avaliados, bem como reconhecer os aspectos frágeis de cada um nos referidos serviços, para que se possa intervir de forma mais efetiva e direcionada.

Nessa direção, apontamos a necessidade de repensar o atendimento oferecido pelas unidades de saúde, priorizando o foco no estabelecimento dos atributos da atenção primária em todas as direções, tornando-a, dessa forma, fonte segura de atendimento à saúde da criança e promovendo a melhoria dos serviços de saúde pública no município.

br/2013/vol46n4/AO_a\%20Longitudinalidade\%20 e\%20integralidade\%20no\%20cuidado\%20a\%20menores\%20de\%20um\%20ano.pdf $>$. Acesso em: 21 jan. 2013.

BRASIL. Ministério da Saúde. Carta dos direitos dos usuários da saúde. Brasília, DF: MS, 2006. Disponível em: <http://conselho.saude.gov.br/biblioteca/livros/ cartaaosusuarios01.pdf $>$. Acesso em: 18 abr. 2012.

Ministério da Saúde. Secretaria de Atenção em Saúde. Departamento de Atenção Básica. Manual do instrumento de Avaliação da Atenção Primária em Saúde: Primary Care Assessment Tool. PCATool Brasil. Brasília, DF: MS, 2010.

COSTA, G. D. et al. Avaliação da atenção à saúde da 
criança no contexto da Saúde da Família no município de Teixeiras, Minas Gerais. Rev. Ciência \&t Saúde Coletiva, Rio de Janeiro, v. 16, n. 7, jul. 2011, p. 32293240. Disponível em: <http://www.scielo.br/pdf/csc/ vl6n7/22.pdf>. Acesso em: 10 jul. 2013.

CUNHA, E. M.; GIOVANELLA, L. Longitudinalidade/ continuidade do cuidado: identificando dimensões e variáveis para a avaliação da Atenção Primária no contexto do sistema público de saúde brasileiro. Rev. Ciência Et Saúde Coletiva, Rio de Janeiro, v. 16, n.1, supl. 1, 2011, p. 1029-1042. Disponível em: <http://www.scielo.br/pdf/ csc/v16sl/a36vl6sl.pdf >. Acesso em: 12 jan. 2013.

GIOVANI, M. S. P.; VIEIRA, C. M. Longitudinalidade do cuidado diante da rotatividade de profissionais na Estratégia Saúde da Família. RECIIS Rev. Eletr. de Com. Inov. Saúde, Rio de Janeiro, v. 7, n. 4, dez. 2013.

GOMES, F. M.; SILVA, M. G. C. Programa Saúde da Família como estratégia de atenção primária: uma realidade em Juazeiro do Norte. Rev. Ciência \&t Saúde Coletiva, Rio de Janeiro. v. 16, supl. 1, 2011, p. 893-902. Disponível em: <http:// www.scielo.br/scielo.php?script=sci_arttext\&pid =S1413-81232011000700021>. Acesso em: 19 jun. 2013.

MELLO, D. F.; FURTADO, M. C. C.; FONSECA, L. M. M.; PINA, J. C. Seguimento da saúde da criança e a longitudinalidade do cuidado. Rev. Bras. Enferm., Brasília, DF, v. 65, n. 4, jul./ago. 2012, p. 675-9. Disponível em: $<$ http://www.scielo.br/pdf/reben/v65n4/a18v65n4. pdf $>$. Acesso em: 8 maio 2013.

MENDES, E. V. As redes de atenção à saúde. Brasília, DF: Organização Pan-Americana da Saúde, 2011.

RIBEIRO, L. C. C.; ROCHA, R. L.; JORGE, M. L. R. Acolhimento às crianças na atenção primária à saúde: um estudo sobre a postura dos profissionais das equipes de saúde da família. Cad. Saúde Pública, Rio de Janeiro, v. 26, n. 12, dez. 2010, p. 2316-2322. Disponível em: <http://www.scielo.br/scielo.php?script=sci arttext\&pid=S0102-311X2010001200010 $>$. Acesso em: 20 maio 2013

ROECHER, S.; BUDÓ, M. L. D.; MARCON, S. S. Trabalho Educativo do enfermeiro na Estratégia Saúde da Família: dificuldades e perspectivas de mudanças. Rev. Esc. Enferm. USP, São Paulo, v.46, n.3, jun. 2012, p. 641-9. Disponível em: <http://www.scielo.br/pdf/ reeusp/v46n3/16.pdf >. Acesso em: 9 jan. 2013.

SILVA, R. M. M. et al. Resolutividade na atenção à saúde da criança: percepção de pais e cuidadores. Acta Paul Enferm, São Paulo, v.26, n.4, 2013, p.382-388. Disponível em: <http://www. scielo.br/scielo.php?script=sci_arttext\&pid $=$ S0103-21002013000400013 $>$. Acesso em: 16 mar. 2014.

STARFIELD, B. Atenção primária: equilíbrio entre necessidades de saúde, serviços e tecnologia. Brasília: UNESCO/Ministério da Saúde, 2002. Disponível em: $<$ http://www.livrosgratis.com.br/arquivos_livros/ ue000039.pdf > . Acesso em: 18 abr. 2012.

VIACAVA, F. et al. Avaliação de desempenho de Sistemas de Saúde: um modelo de análise. Rev. Ciência \& Saúde Coletiva, Rio de Janeiro, v. 17, n. 4, abril 2012, p. 921-934. Disponível em: <http:// www.scielosp.org/scielo.php?script=sci_arttext\&pi $\mathrm{d}=$ S1413-81232012000400014>. Acesso em: 29 mar. 2013.

Recebido para publicação em abril de 2014 Versão final em novembro de 2014 Conflito de interesse: inexistente

Suporte financeiro: Recorte de pesquisa financiada pelo CNPq, Edital Universal 014/2012, processo ํㅜ 474743/2011-0 